ORIGINAL ARTICLE

\title{
Strength performance parameters when adopting different exercise sequences during agonist-antagonist paired sets
}

\author{
Marianna de Freitas Maia, Gabriel Andrade Paz, João Souza, Humberto Miranda*
}

Federal University of Rio de Janeiro, School of Physical Education and Sports, RJ, Brazil

Received 14 September 2014; accepted 12 January 2015

Available online 13 May 2015

\section{KEYWORDS \\ Resistance training; \\ Strength \\ performance; \\ Electromyography}

\begin{abstract}
Objective: To investigate the effect of different exercise sequences during agonist-antagonist paired sets on training volume, ratings of perceived exertion, and muscle activation.

Material and methods: Thirteen recreationally trained males participated in this study. Two protocols were adopted in two non-consecutive days. BS - participants performed three repetition to failure sets (with 8 repetition maximum loads) of bench-press (BP) followed by seated row exercise in alternate manner. $S B$ - the seated row (SR) was performed before bench press. Two-minute rest interval was adopted between sets and exercises. The number of repetitions and electromyography signals of the posterior deltoids (PD), biceps brachii (BB), pectoralis major (PM), and triceps brachii (TB) muscles were recorded during both exercises.

Results: No significant differences were noted in training volume $(1486.6 \pm 200.3 ; 1492 \pm 282.5)$ and total work $(22.3 \pm 1.3 ; 22 \pm 2)$ BS and SB sequences for BP, respectively. Higher training volume $(1709.7 \pm 177.6 ; 1424.4 \pm 196)$ and total work $(25.3 \pm 1.8 ; 21 \pm 1.6)$ were noted for SR under BS compared to SB. Higher PD activation was noted during SR under BS compared to SB. Conclusion: The exercise sequence showed significant differences in strength performance and agonist muscle activation during agonist-antagonist paired sets for SR exercise. These results suggest that antagonist pre-loading may have a potential effect on back muscles.

() 2014 Consell Català de l'Esport. Generalitat de Catalunya. Published by Elsevier España, S.L.U. All rights reserved.
\end{abstract}

\footnotetext{
* Corresponding author.

E-mail address: humbertomirandaufrj@gmail.com (H. Miranda).
} 


\section{PALABRAS CLAVE \\ Entrenamiento de \\ fuerza; \\ Desempeño de \\ fuerza; \\ Electromiografía}

Parámetros de rendimiento de fuerza mediante la adopción de diferentes secuencias de ejercicios durante series emparejadas agonista-antagonista

\begin{abstract}
Resumen
Objetivo: Investigar el efecto de distintas secuencias de ejercicios de series emparejadas de los músculos agonistas/antagonistas sobre el volumen del entrenamiento, la percepción subjetiva del esfuerzo y la activación muscular.

Material y método: Trece deportistas de recreación, hombres, fueron voluntariamente sometidos a este estudio. Se aplicaron 2 protocolos en 2 días no consecutivos. Protocolo BR: los participantes realizaron 3 series de ejercicios de press de banca (PB) hasta el agotamiento (con carga de 8 repeticiones máximas) seguidas del ejercicio de remo sentado (RS), alternadamente. Protocolo RB: el remo sentado se realizó antes del PB. Se adoptó un intervalo de recuperación de $2 \mathrm{~min}$. Entre repeticiones y ejercicios. Durante los 2 ejercicios se anotaron el número de repeticiones y la señal electromiográfica de los músculos deltoides posterior (DP), del bíceps braquial (BB), del pectoral mayor (PM) y del tríceps braquial (TB).

Resultado: No se encontraron diferencias significativas en el volumen de entrenamiento $(1.486,6 \pm 200,3 ; 1.492 \pm 282,5)$ y del trabajo total $(22,3 \pm 1,3 ; 22 \pm 2)$ entre las secuencias $B R$ y RB, para PB, respectivamente. Se observó un volumen de entrenamiento $(1.709,7$ $\pm 177,6 ; 1.424,4 \pm 196)$ y del trabajo total $(25,3 \pm 1,8 ; 21 \pm 1,6)$ superiores en el RS en la secuencia BR, comparado con RB.

Conclusión: La secuencia del ejercicio mostró diferencias significativas en el rendimiento de fuerza y activación muscular de los agonistas durante las series emparejadas agonistaantagonista en el ejercicio de RS. Estos resultados indican que la precarga de los antagonistas puede generar un efecto potencial para favorecer el rendimiento de los músculos dorsales. (c) 2014 Consell Català de l'Esport. Generalitat de Catalunya. Publicado por Elsevier España, S.L.U. Todos los derechos reservados.
\end{abstract}

\section{Introduction}

Resistance training has been widely used as an effective method for developing strength, endurance and/or power performance for athletes or general population. ${ }^{1}$ The manipulation of intensity and volume of training are essential to increase the outcomes during resistance training programs. ${ }^{2-4}$ Training volume (sets $\times$ repetitions $\times$ external load) is usually calculated by coaches and athletes during the resistance training sessions in order to increase the strength gains in acute and/or chronic manner. ${ }^{5}$

Several training systems are often applied in order to manipulate the training volume. One of these training systems is the agonist-antagonist paired set (PS), which can be accomplished in a shorter time (efficiency) without compromising efficacy (training volume). ${ }^{6}$ PS training is characterized by performing exercises for agonist and antagonist muscles in alternated manner, with or without rest intervals between sets and exercises. ${ }^{7}$ Previous evidences have been suggested that PS training allows a similar or higher strength performance than traditional set (TS) training, with a significant reduction in the duration of training session. ${ }^{6,8,9}$

Robbins et al. ${ }^{7}$, observed similar training volume between TS (with 4-minute rest interval between sets and exercises) and PS (with 2-minute rest interval) training for bench pull and bench press exercises, with four repetition maximum (RM) loads. However, Robbins et al. ${ }^{7}$, noted a higher efficiency (training volume/time) under PS, compared to TS considering training session duration. Recently, Maia et al. ${ }^{9}$ found significant increases on repetition performance and electromyographic (EMG) data of vastus medialis and rectus femoris during leg extension followed by lying leg curl exercise (with 10RM loads), compared to a set of leg extension exercise performed without antagonist preloading. Similar results were noted by Paz et al. ${ }^{10}$, who noted higher repetition performance and muscle activation of latissimus dorsi and biceps brachii for seated row exercise (10RM loads) immediately after a of bench press, when compared to set of seated row without antagonist preloading. Considering the effect of exercise order during APS, Balsamo et al. ${ }^{8}$, observed higher training volume and lower ratings of perceived exertion (RPE) under PS session with leg curl followed by leg extension resistance exercise (with 10RM loads), compared to leg extension performed before leg curl exercise. These data suggest that the exercise order during PS training may promote significant interference on strength performance.

Several studies have shown that exercise order promote significant impact on repetition performance in acute manner during resistance training programs. ${ }^{3,4,11}$ However, the effect of exercise order during PS training on muscles activation and strength performance are still unclear. These evidences may be useful resistance training practitioners and coaches who want to improve the strength outcomes and also reduce the training session duration. Therefore, the purpose of this study was to investigate the effect of PS training compared to TS on training volume, RPE and muscle 
activity of pectoralis major, biceps brachii, triceps brachii and posterior deltoid.

\section{Materials and methods}

\section{Participants}

Thirteen recreationally trained men were recruited from a local university using convenience sampling, with the following mean ( \pm standard deviation, SD) characteristics: age $=22.1 \pm 1.2$ years, height $=173 \mathrm{~cm} \pm 5.2$, weight $=74.6 \mathrm{~kg} \pm 5.1$ and body fat percentage $=11.4 \% \pm 2.1 ;$ resistance training experience $=3.1 \pm 1$ years. The inclusion criteria were: To have at least one year of resistance training experience, with averaging four 60 -minute sessions per week using 1- to 2-minute rest intervals between sets and exercises. The exclusion criteria were: To have any functional limitation or medical condition that could influence their ability to perform the testing or experimental protocols were excluded from the current study and to perform upper-body exercise in the 48 hours prior to each session. The current study was approved by the Institutional Human Experimental Committee at University. Written informed consent was obtained from all participants prior to participation, in accordance with the Declaration of Helsinki.

\section{Procedures}

The current study employed a randomized crossover design comprised of 4 visits carried out on nonconsecutive days with $48-72 \mathrm{~h}$ of rest interval. All tests were completed at the same time of day, and the participants were instructed to avoid any type of exercise during the period of the test sessions. The first two testing sessions focused on measures of strength and anthropometry. Anthropometric data were measured following the protocol of Jackson and Pollock. ${ }^{12}$

\section{Eight repetition maximum tests (8RM)}

At each of these sessions, strength was assessed using an 8RM test for bench-press and wide-grip seated row exercises on machines (Life Fitness, Rosemont, IL, USA). The 8RM test was performed at a constant pace (two seconds for concentric and two seconds for eccentric actions) and was controlled by a metronome (Metronome Plus 2.0, M\&M Systeme Germany). ${ }^{13}$ If the participant did not attain 8 repetitions in the first attempt, the weight was adjusted by $4-10 \mathrm{~kg}$, and a minimum of five minutes of rest was given before the next attempt. Ten-minute rest intervals were adopted between exercises to test the loads for 8RM. Bench-press and seated row exercises were alternated during test and retest. Only three trials were allowed per testing session. The test and retest sessions were conducted with 48 hours of intervals (Fig. 1).

\section{Exercise sessions}

At the third and fourth visits, participants were assigned to exercise sequences in a randomized fashion: BS - participants performed three paired sets repetition to failure of bench-press followed by seated row exercise with 8RM loads, adopting two-minute rest interval between sets and exercises; SB - participants performed three paired sets repetition to failure of seated row followed by bench-press exercise with 8RM loads, adopting two-minute rest interval between sets and exercises. Before each protocol, participants performed a warm-upset of 15 repetitions using $50 \%$ of 8RM loads for the first exercise performed, followed by a 2-minute rest interval before beginning the experimental protocols. ${ }^{14}$ The number of repetitions and electromyographic (EMG) activity of the posterior deltoids (PD), biceps brachii (BB), pectoralis major (PM), and triceps brachii (TB) muscles were assessed during the seated row and benchpress exercises in each protocol.

\section{Perceived exertion assessment procedures}

During the experimental protocols, the participants were provided perceived exertion scaling instructions and anchoring procedures for the OMNI-RES before exercising. ${ }^{15}$ The instructions also explained the nature and use of the OMNIRES, differentiated ratings and how to use the low and high numerical categories as scale anchor points. The scale anchoring procedure provided the subject with an understanding of the range of perceptions that corresponds to the low- and high-rating categories. The anchoring procedures allowed the subjects to experience the 2 extremes of RPE: rating 1 (extremely light) and rating 9 (extremely hard). In our study, the anchoring of rating was providing at the end of each set performed in bench-press and seated row exercise.

\section{Surface electromyography}

The EMG signal was captured through passive bipolar surface electrodes (Kendal Medi Trace 200, Tyco Healthcare, Pointe-Claire, Canada), acquired by a dedicated data acquisition system model PS850 (Biometrics, Newport, UK). The signals were amplified by 1000 (CMRR $>100 \mathrm{~dB}$ ), and sampled at $1000 \mathrm{~Hz}$ after being band-pass filtered $(10-500 \mathrm{~Hz})$. Some precautions were taken in order to avoid the dynamic EMG limitations, such as the placement and location of the electrodes which was made in accordance with surface electromyography for the non-invasive assessment of muscles (SENIAM) recommendations. ${ }^{16}$ Skin surface was shaved, slightly abraded, and cleaned with alcohol swabs before placing the EMG surface electrodes. These were placed on the corresponding muscle belly aligned with the fiber direction, according to SENIAM standards, in order to avoid the possibility of crosstalk. ${ }^{17}$ The PM electrode was placed at the midpoint between the acromion process and the xiphoid process. The PD electrode was in the area about two finger breaths behind the angle of the acromion. The BB electrode was placed on the line between the medial acromion and the cubit fossa. The TB electrode was placed half way between the acromion process and the olecranon process at 2 finger widths below the medial line. The reference electrode was 
BS

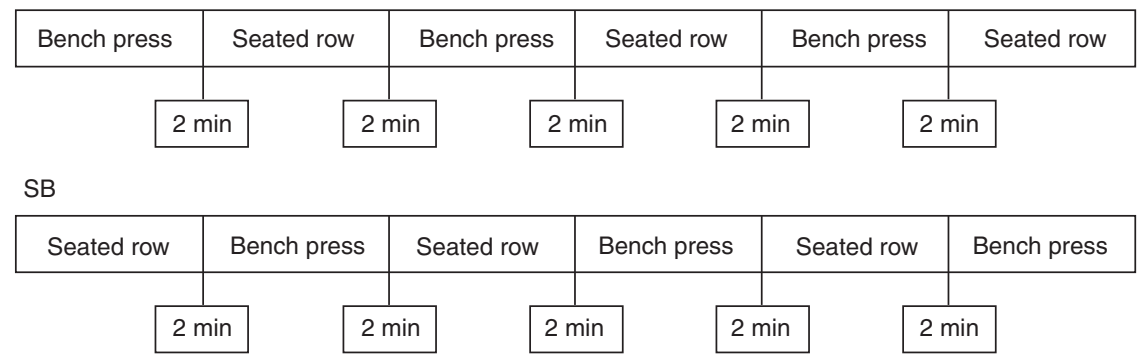

Figure 1 Study design.

placed in the clavicle bone. The impedance between electrode pairs was less than $5 \mathrm{k} \Omega$ using a $25-\mathrm{Hz}$ signal through the electrodes. All these procedures were performed by the same investigator. Placement of the electrodes was identified on the first day of testing, and an indelible pen mark was made on the skin to ensure that the same position was used on subsequent days.

\section{Data analysis}

The mean amplitude of the root mean square (RMS) was assessed using the custom-written software Matlab 5.02c (MathWorks TM, Natick, MA, USA). The averaging window for the RMS was 100 milliseconds, and all reported values are the mean RMS over a predetermined sampling window from the onset to the end of each contraction. Only the signal of central repetitions obtained was analyzed, excluding the first and last repetition of each set and exercise. This procedure was adopted to avoid problems with signal discrepancies regarding the inertia at the beginning of exercises, as well as the possibility of fatigue in the last repetition. EMG data were collected for the entire (concentric and eccentric phases) for each set, exercise and protocol. EMG data were expressed as a percentage relative to the largest RMS value $(100 \%)$ of the EMG signal obtained for each muscle considering all sets and protocols performed respectively. ${ }^{18}$ The normalization via the peak of EMG data obtained during all sets and exercise for each muscle was adopted to avoid and attenuate some limitations of EMG analysis during these dynamic tasks such as changes in either the shape of the intracellular action potential, volume conductor, sarcolemmal properties of the muscle fibers, or differences in subcutaneous tissue thickness. $^{17}$

\section{Statistical analyses}

The statistical analyses included the test-retest reliability of $8 R M$ loads and EMG spectral parameters using the intraclass correlation coefficient $(I C C=(M S b-M S W) /[M S b+(k-1) M S W)])$, where $M S b=$ meansquare between, $M S w=$ means-square within, and $\mathrm{k}=$ average group size. The Shapiro-Wilk test and homoscedasticity (Bartlett criterion) showed that all variables presented normal distribution and homoscedasticity. These data were analyzed using a 2-way analysis of variance
(ANOVA) $(2$ [sequences] $\times 3$ [sets]) with repeated-measures to determine whether there were significant main effects or interactions between the exercise sequences and the sets $(1,2$, and 3$)$. EMG data were analyzed using a 3-way ANOVA ( 2 [sequence] $\times 3$ [sets] $\times 4$ [muscles]) with repeated measures to determine whether there were significant main effects or interactions among the exercise sequences, sets $(1,2$, and 3$)$ and muscles analyzed. Post hoc tests with the Bonferroni correction were employed when necessary. Paired $T$ test was adopted to compare the total work (sum of the number of repetitions over the three sets) between the protocols for each exercise. To verify the differences in the total RPE between protocols and exercises, Wilcoxon test was used, respectively. The level of statistical significance was set at 0.05 for all tests. The effect size was also computed following the Rhea $(2004)^{19}$ recommendations. The statistical analysis was performed with SPSS version 20.0 (Chicago, IL, USA).

\section{Results}

There was no significant differences between sequences $(F=0.013 ; p=0.912)$ for bench-press exercise, however, significant differences were noted between sets $(F=77.775 ; p=0.0001)$ (Table 1$)$. Thus, significant interactions were observed between sets and sequences $(F=10.191 ; p=0.001)$. On the other hand, there were no differences on training volume and total work between sequences for bench press exercise. Considering the seated row exercise, there was significant differences between sequences $(F=40.904 ; p=0.0001)$ and sets $(F=48.941$; $p=0.0001)$. Thus, there was no interaction between sets and sequences $(F=1.807 ; p=0.186)$. However, higher training volume $(p=0.0001)$ and total work $(p=0.0001)$ were noted for seated row exercises, under BS sequence compared to SB.

The RPE was significantly higher from set $2(p=0.002$; $p=0.0001)$ and $3(p=0.003 ; p=0.0001)$ to set 1 , under BS and SB sequences for bench press exercise, respectively (Fig. 2 ). Thus, the RPE was greater during set 3 under SB $(p=0.0001)$ compared to BS sequence. However, no significant difference was found in the RPE for seated row exercise over the three sets for both sequences (Fig. 3).

There was no significant difference or interaction between sets and sequence for TB and PM muscles activation during bench-press exercise. This was also true for $\mathrm{BB}$ activation during bench-press exercise. However, there 
Table 1 Mean, SD and effect size of repetition performance, training volume and total work for bench press and seated row during the experimental protocols.

\begin{tabular}{llllll}
\hline & Set 1 & Set 2 & Set 3 & TV & TW \\
\hline $\begin{array}{lllll}\text { Bench press } \\
\text { BS }\end{array}$ & $8 \pm 0$ & $7.6 \pm 0.7$ & $6.6 \pm 0.8 \# \S$ & $1486.6 \pm 200.3$ & $22.3 \pm 1.3$ \\
SB & $9 \pm 1$ & $7 \pm 0.6 \#$ & $6.1 \pm 0.8 \# \S$ & $1492 \pm 282.5$ & $22 \pm 2$ \\
$\quad$ Effect size & 1 (moderate) & -0.85 (trivial) & -0.62 (trivial) & 0.02 (trivial) & -0.23 (trivial) \\
Seated row & & & & \\
BS & $9.2 \pm 1.4^{*}$ & $8.3 \pm 0.6^{*}$ & $7 \pm 0.8 \# \S^{*}$ & $1709.7 \pm 177.6^{*}$ & $25.3 \pm 1.8^{*}$ \\
SB & $7.9 \pm 0.2$ & $7 \pm 0.8$ & $6.1 \pm 1.1 \# \S$ & $1424.4 \pm 196$ & $21 \pm 1.6$ \\
Effect size & -0.92 (trivial) & -2.1 (trivial) & -1.12 (trivial) & -1.60 (trivial) & -2.38 (trivial) \\
\hline
\end{tabular}

BS: bench press followed by seated row exercise; SB: seated row followed by bench press exercise; TV: training volume (repetition $\times$ sets $\times$ external load); TW: total work (repetitions $\times$ sets); * significant difference to SB sequence; $¥$ significant difference to BS sequence; \# significant difference for set 1 ; $\S$ significant difference for set 2.

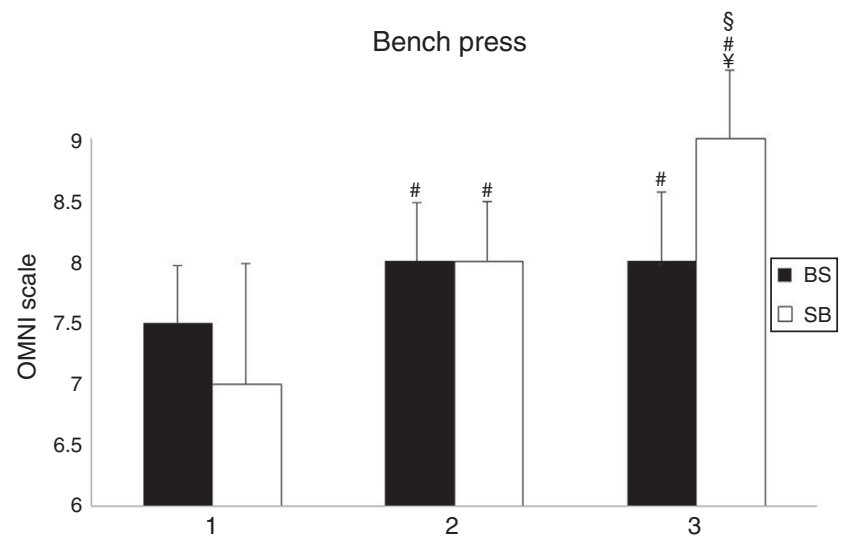

Figure 2 Ratings of perceived exertion values (median) following the OMNI-RES scale for bench press exercise. BS: paired sets between bench press followed by seated row exercise; SB: paired sets between seated row followed by bench press exercise; * significant difference for SB sequence; ¥ significant difference for BS sequence; \# significant difference for set $1 ; \S$ significant difference for set 2 .

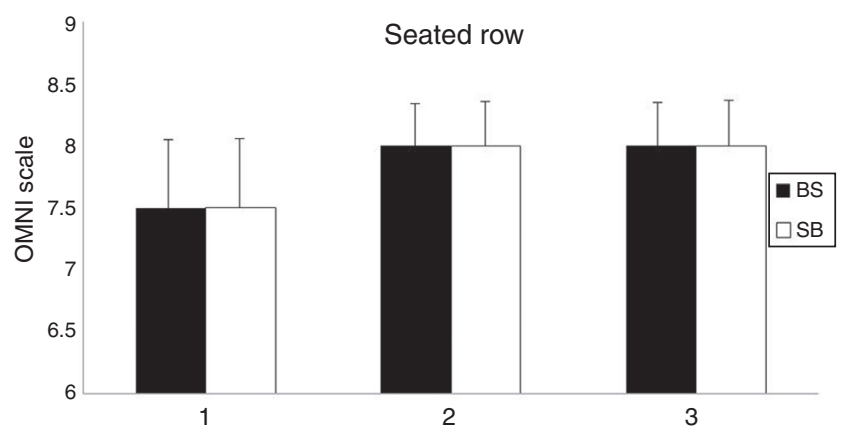

Figure 3 Ratings of perceived exertion values (median) following the OMNI-RES scale for seated row exercise. BS: paired sets between bench press followed by seated row exercise; $\mathrm{SB}$ : paired sets between seated row followed by bench press exercise.

was significant difference between protocols for PD muscle activation $(F=5.454 ; p=0.038)$. The PD coactivation was significantly higher during set $1(p=0.002), 2(p=0.0001)$ and $3(p=0.0001)$ under BS compared to SB sequence (Fig. 4).
There was significant difference between protocols for PD muscle $(F=6.324 ; p=0.021)$. The PD coactivation was significantly higher during set $1(p=0.021), 2(p=0.001)$ and $3(p=0.0001)$ under BS compared to SB condition. There was no significant difference or interaction between sets and protocols for BB muscles during seated row exercise for both sequences. Similar results were found for TB activation during seated exercise (Fig. 5).

\section{Discussion}

The main findings of this investigation demonstrate that there was significant higher training volume for seated row exercise when it was performed after bench press exercise, compared to inverse order during PS training. Higher PD muscle activation was also noted over the three sets performed for seated row, under BS protocol, compared to $\mathrm{SB}$. These results are in agreement with previous evidences which found significant improvements on strength performance following antagonist pre-loading protocols. ${ }^{8,20}$ These findings may also indicate that the exercise order should be considered during the prescription of PS training.

Higher training volume was observed in the current study for seated row exercise when it was performed after bench press exercise (BS), compared to inverse order (SB). These results were similar to those found by Balsamo et al. ${ }^{8}$, who noted significantly higher training volume when leg extension exercise was performed after leg curl exercise (10RM loads), compared to the inverse order (leg extension performed before leg curl exercise). Balsamo et al. ${ }^{8}$, suggests that the hamstring muscles were more sensitive to alterations on muscles spindles, Golgi tendon organs and elastic energy storage compared to quadriceps muscles, which may be responsible for the potential effect of antagonist preloading. Considering the seated row exercise, Paz et al. ${ }^{10}$ observed higher repetition performance after an antagonist preloading set of bench press (10RM loads), when compared to a set of seated row exercise performed without antagonist preloading.

However, in the current study, there was no significant difference on training volume and total work for bench press exercise between both sequences. This was also true for RPE between sequences and exercises. Similar results were 

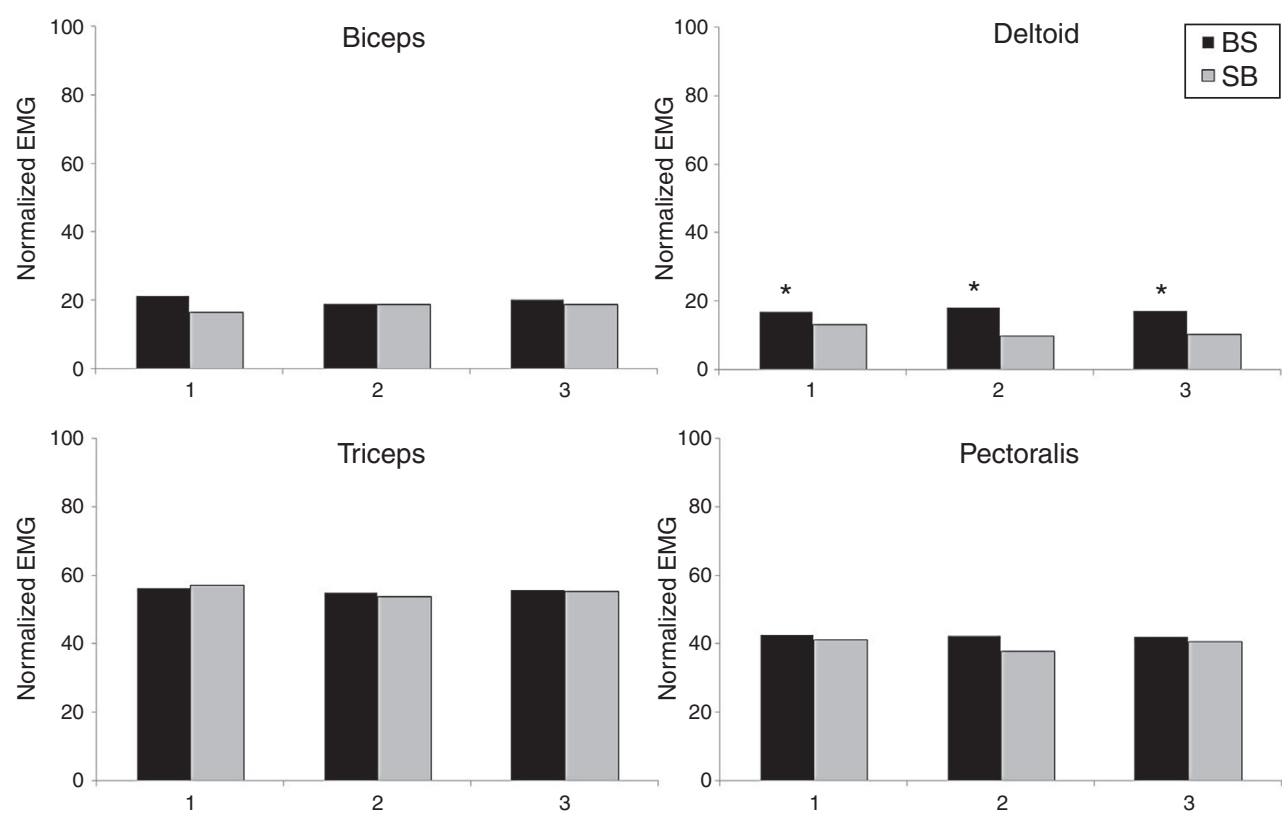

Figure 4 Mean normalized root mean square of biceps brachii, posterior deltoid, pectoralis and triceps brachii muscles during bench press exercise. *Significant difference for SB protocol $(p<0.05)$.

noted by Robbins et al. ${ }^{7}$, who found similar training volume under three of PS for bench pulls and bench press (4RM loads) exercises, using 4-minute rest intervals between sets and exercises, compared TS training. Robbins et al. ${ }^{7}$ suggest that the back muscles are more resistant to accumulate fatigue, which may affect the antagonist preloading adjustment. In another study, Robbins et al. ${ }^{6}$ also observed similar training volume following three PS (2-minute rest interval) of bench pulls and bench press (4RM), compared to TS training (4-minute rest interval). These results were different to those observed by Baker and Newton ${ }^{20}$, who noted higher power performance during bench press throws 3-minute after a set of bench pulls exercise, compared to bench press throws without antagonist preloading. Baker and Newton ${ }^{20}$ suggested that this increasing on agonist power performance was due to an alteration on triphasic pattern of activation (agonist-antagonist-agonist) induced by antagonist preloading.

Higher muscle activation was noted for PD muscles over the three sets performed for seated row exercise under
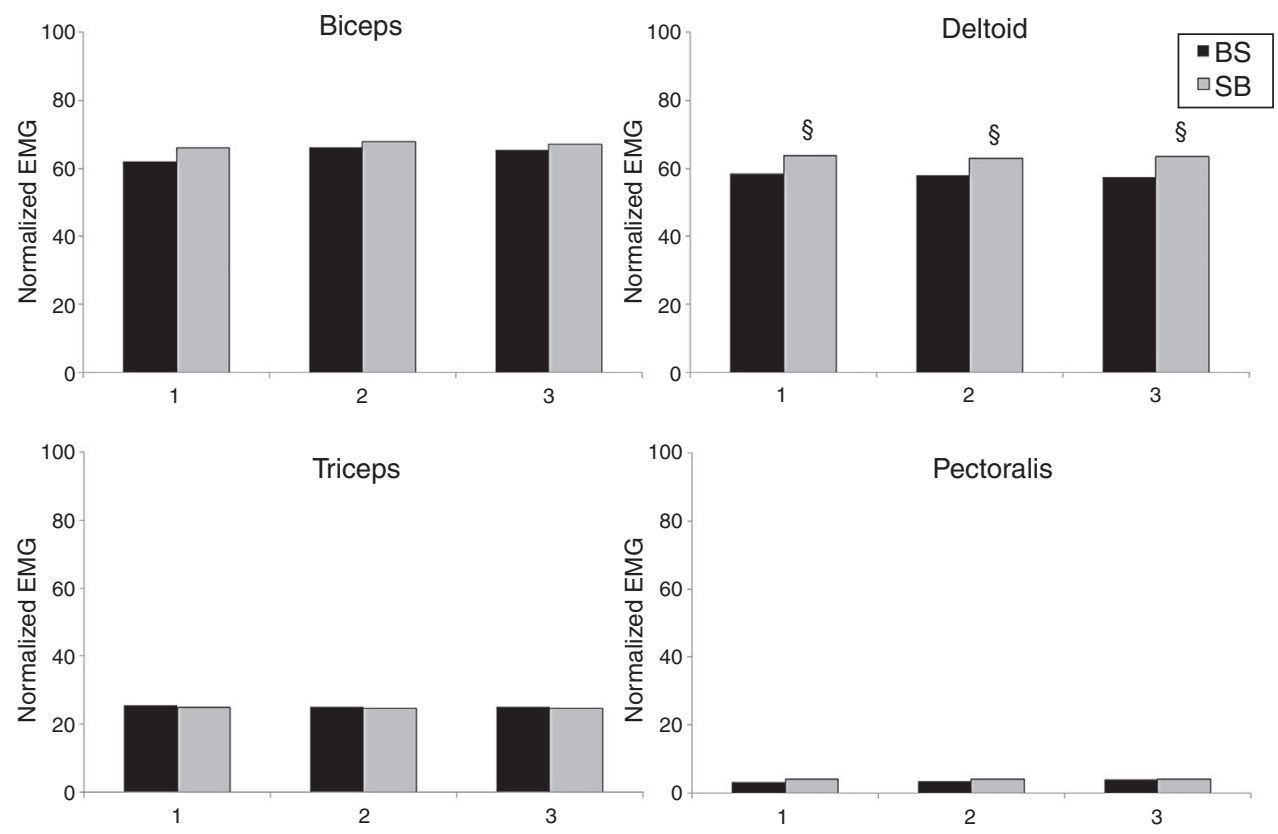

Figure 5 Mean normalized root mean square of biceps brachii, posterior deltoid, pectoralis and triceps brachii muscles during seated row exercise. $¥$ Significant difference for BS protocol $(p<0.05)$. 
BS sequence, compared to SB sequence. These results are in disagreement with previous researchers which did not found difference on agonist or antagonist muscle activation between PS training compared to TS training. ${ }^{7,6}$ However, similar results were observed by Paz et al. ${ }^{10}$, who noted higher muscle activation for latissimus dorsi and BB during seated row followed by a set of bench press, compared to a set without antagonist preactivation. The authors associated these results to a fatigue state induced by antagonist manipulation. Recently, Maia et al. ${ }^{9}$, observed significant increases on vastus medialis and rectus femoris muscle activation during a set of leg extension exercise (10RM loads) followed by lying leg curl exercise, adopting limited or shorter rest intervals (30 s and 1-minute), compared to a protocol without antagonist preloading. These results supports the hypotheses which indicate that antagonist preactivation could improve the agonist muscle activation and strength performance. ${ }^{6}$

In the present study, significant increases on PD activation were noted during bench press exercise over the three sets, under BS sequence compared to SB. This results may be associated to shoulder stabilizing function of PD muscles during seated row exercise (shoulder abduction), considering the accumulated fatigue over the PS, and increasing in the number of repetitions performed under BS sequence. The increased EMG amplitude observed during BS might be primarily attributed to additional motor unit recruitment and/or increased spatial or temporal motor unit synchronization, presumably to compensate for muscle fiber fatigue. ${ }^{21,22}$ Several mechanisms (e.g., neural adjustment of Golgi tendon organ, increased elastic energy storage, alteration of triphasic neural pathways) have been proposed to explain antagonist-pre-load induced performance. ${ }^{7,8,20,23}$ On the other hand, the alteration on triphasic pattern may not be responsible for the results found in the current study, considering the triphasic pattern of activation are often induced by higher speed movements.

A secondary finding of the present study was the observed decreases in repetition performance for both bench press and seated row across sets under both protocols. These data suggest that a 2 -min rest interval was inadequate to maintain repetition performance. This finding is consistent with previous PS research in which repetition performance was not maintained when using rest intervals of $1-4$ minutes between like exercise sets. 3,6,24

This study has limitations that warrant mentioning. Due to factors such as muscle speed, fiber and length, the interpretation of the EMG signal during dynamic tasks may increase the non-stationary characteristics of the EMG signal. Additionally, the current study only examined two upper body resistance exercises, whereas resistance training sessions typically include various exercises performed over multiple sets. Thus, the interaction between agonist and antagonist muscles has a greater potential and practical applicability for acute improvements on strength performance which should be explored in future researchers.

\section{Conclusion}

The results of the current study suggests that the exercise order has a greater effect on strength performance during
PS training for training volume, total work and muscle activation, considering upper body muscles. If the goal of the training session is to increase the repetition performance and muscles activation in acute manner, the bench press exercise might be performed before seated row exercise.

\section{Conflict of interest}

The authors have no conflict of interest.

\section{Acknowledgements}

The authors thank the Education Program for Work and Health (PET-SAUDE) and also the Coordination of Improvement of Higher Education Personnel (CAPES/Brazil) for the master's scholarship conceded to G.A. Paz.

\section{References}

1. American College of Sports Medicine (ACSM). Position Stand: quantity and quality of exercise for developing and maintaining cardiorespiratory, musculoskeletal, and neuromotor fitness in apparently healthy adults: guidance for prescribing exercise. Med Sci Sports Exerc. 2011;43:1334-59.

2. American College of Sports Medicine (ACSM) Position Stand: Progression Models in Resistance Training for Healthy Adults. Med Sci Sports Exerc. 2009;34:687-708.

3. Miranda H, Simao R, dos Santos Vigario P, de Salles BF, Pacheco MT, Willardson JM. Exercise order interacts with rest interval during upper-body resistance exercise. J Strength Cond Res. 2010;24:1573-7.

4. Simao R, de Salles BF, Figueiredo T, Dias I, Willardson JM. Exercise order in resistance training. Sports Med. 2012;42:251-65.

5. Fleck SJ, Kraemer WJ. Designing resistance training programs. Fourth Edition Human Kinects; 2014.

6. Robbins DW, Young WB, Behm DG, Payne WR. Agonist-antagonist paired set resistance training: a brief review. J Strength Cond Res. 2010;24:2873-82.

7. Robbins DW, Young WB, Behm DG. The effect of an upper-body agonist-antagonist resistance training protocol on volume load and efficiency. J Strength Cond Res. 2010;24:2632-40.

8. Balsamo S, Tibana RA, NascimentoDda C, de Farias GL, Petruccelli Z, de Santana Fdos, et al. Exercise order affects the total training volume and the ratings of perceived exertion in response to a super-set resistance training session. Int J Gen Med. 2012;5:123-7.

9. Maia MF, Willardson JM, Paz GA, Miranda H. Effects of different rest intervals between antagonist paired sets on repetition performance and muscle activation. J Strength Cond Res. 2014;28:2529-35.

10. Paz GA, Willardson JM, Simão R, Miranda H. Effects of different antagonista protocols on repetition performance and muscle activation. Med Sport. 2013;17:106-12.

11. Miranda H, Figueiredo T, Rodrigues B, Paz GA, Simão R. Influence of exercise order on repetition performance among all possible combinations on resistance training. Res Sports Med. 2013;21:355-66.

12. Jackson AS, Pollock ML. Generalized equations for predicting body density of men. Br J Nutr. 1978;40:497-504.

13. Gentil PE, Oliveira E, de Araújo Rocha Júnior V, do Carmo $J$, Bottaro M. Effects of exercise order on upper-body muscle activation and exercise performance. J Strength Cond Res. 2007;21:1082-6. 
14. Tan B. Manipulating resistance training program variables to optimize maximum strength in men: a review. J Strength Cond Res. 1999;13:289-304.

15. Robertson RJ, Goss FL, Rutkowski J, Lenz B, Dixon C, Timmer $\mathrm{J}$, et al. Concurrent validation of the OMNI perceived exertion scale for resistance exercise. Med Sci Sports Exerc. 2003;35:333-41.

16. Hermens HJ, Freriks B, Disselhorst-Klug C, Rau G. Development of recommendations for SEMG sensors and sensor placement procedures. J Electromyogr Kinesiol. 2000;10:361-74.

17. De Luca CJ. The use of surface electromyography in biomechanics. J Appl Biomech. 1997;13:135-63.

18. Wright GA, Delong TH, Gehlsen G. Electromyographic activity of the hamstrings during performance of the leg curl, stiffleg deadlift, and back squat movements. J Strength Cond Res. 1999;13:168-74.

19. Rhea M. Determining the magnitude of treatment effects in strength training research through the use of the effect size. J Strength Cond Res. 2004;18:918-20.
20. Baker D, Newton RU. Acute effect on power output of alternating an agonist and antagonist muscle exercise during complex training. J Strength Cond Res. 2005;19:202-5.

21. Benson C, Docherty D, Brandenburg J. Acute neuromuscular responses to resistance training performed at different loads. J Sci Med Sport. 2006;9:135-42.

22. Marshall PW, Robbins DA, Wrightson AW, Siegler JC. Acute neuromuscular and fatigue responses to the rest-pause method. J Sci Med Sport. 2012;15:153-8.

23. Carregaro R, Cunha R, Oliveira CG, Brown LE, Bottaro M. Muscle fatigue and metabolic responses following three different antagonist pre-load resistance exercises. J Electromyogr Kinesiol. 2013;23:1090-6.

24. Robbins DW, Young WB, Behm DG, Payne WR. The effect of a complex agonist and antagonist resistance training protocol on volume load, power output, electromyographic responses, and efficiency. J Strength Cond Res. 2010;24: 1782-9. 